\title{
The Design of the Cooling System for the CMS Barrel Electromagnetic Calorimeter
}

\author{
Olivier Teller, on behalf of the CMS ECAL group
}

\begin{abstract}
CMS is one of the four experiments being constructed for the LHC. The electromagnetic calorimeter (ECAL) is made of Lead Tungstate $\left(\mathrm{PbWO}_{4}\right)$ crystals. Scintillation light from the crystals in the Barrel is detected by Avalanche Photo Diodes (APD). The number of scintillation photons emitted by the crystals and the amplification of the APD are both temperature dependent. Keeping the constant term of the energy resolution (the contribution to the fractional energy resolution which is independent of energy) below a few tenths of percent requires the temperature of the the crystals and APDs to be stabilized to within $0.1 \mathrm{~K}$ while for example previously built $L 3$ detector using another type of crystals had a temperature stability around $1 \mathrm{~K}$ which is the standard requirement for cooling systems at CERN. In this contribution, we describe the innovative solutions which have been developed and implemented to achieve this performance.
\end{abstract}

Index Terms-CERN, CMS, cooling, crystal, ECAL, electromagnetic calorimeter, LHC, particles detector, temperature control.

\section{INTRODUCTION}

$\mathbf{T}$ HE Compact Muon Solenoid (CMS) detector [1] is a general purpose detector to be installed at the $14 \mathrm{TeV}$ proton-proton collider, LHC, under construction at CERN and due to start operation in 2007. The Electromagnetic Calorimeter (ECAL) [2] of the detector is a hermetic homogeneous calorimeter made of 61,200 lead tungstate $\left(\mathrm{PbWO}_{4}\right)$ crystals mounted in the central barrel part, closed by 7324 crystals in each of the two end-caps.

The barrel is made up of 36 supermodules (SM), each with 1700 crystals arranged in a quasi-projective geometry. Avalanche photodiodes (APDs) are used as photodetectors in the barrel and vacuum phototriodes in the end-caps. One of the driving criteria in the design was the capability to detect the decay to two photons of the postulated Higgs boson. This capability is enhanced by the superior energy resolution provided by a homogeneous crystal calorimeter. One of the key requirements, needed to be able to realise the potential excellent energy resolution in operation, is stability of the calibration and inter-calibration over extended periods of time. The temperature of the crystals needs to be maintained constant to high precision. In addition, the gains of the APDs used in the barrel part are strongly sensitive to both temperature and bias voltage, placing further constraints on thermal stability and power supply regulation. The number of scintillation photons emitted by the crystals and the amplification of the APD are

Manuscript received December 21, 2006; revised June 20, 2007.

The author is with the PH-CMA Group, CERN, CH-1211 Geneva 23, Switzerland (e-mail: Olivier.Teller@cern.ch).

Color versions of one or more of the figures in this paper are available online at http://ieeexplore.ieee.org.

Digital Object Identifier 10.1109/TNS.2007.906169 both temperature dependent. Both variations are negative when the temperature increases. Keeping the constant term of the energy resolution (the contribution to the fractional energy resolution which is independent of energy) below a few tenths of percent requires the temperature of crystals and APDs to be stabilized to within $0.1 \mathrm{~K}$.

In this contribution, we describe the innovative solutions which have been developed and implemented to achieve this performance. We present the precise requirements for every part of the system. We show how the mechanics of the CMS ECAL Barrel has been optimised for thermal performance and describe the design that enables thermal control by water cooling of the supporting structures of both the crystals and the electronics. An important requirement is to allow an easy and reliable mounting of the electronics boards for assembly and maintenance. We also present the design of the cooling plant able to regulate a large flow of coolant ( $62 \mathrm{l} / \mathrm{s}$ water) with the required stability $( \pm 0.025 \mathrm{~K})$.

Several supermodules have already been assembled and tested using a prototype of the final cooling plant. We present the thermal performance measured during these tests, which show that the stability requirements are achieved.

\section{Cooling System ReQuirements}

The cooling specification is driven by the requirement of a $0.5 \%$ constant term in the detector resolution. This leads to the requirement of $0.1 \mathrm{~K}$ peak to peak thermal stability that has to be maintained over several months. However it does not impose any constraints on the absolute temperature nor on the uniformity between any two given crystals. There are two major contributions to temperature fluctuations.

1) The variations of the cooling water temperature at the input of a supermodule.

2) The temperature changes within a supermodule with respect to the cooling water temperature due to the power dissipation of the electronics and the mechanical construction of the supermodules.

As there is no obvious reason why either of the two contributions could be easier or cheaper to minimise, the same stability budget has been given for both leading to the following requirement.

- The water temperature at the inlet of the supermodule should be stable within $0.05 \mathrm{~K}$ peak to peak.

- The crystal and APD temperature stability impact of any change on the electronic dissipation inside the SM should be lower than $0.05 \mathrm{~K}$ peak to peak.

The electronic dissipation inside the SM is around 2.5 W/channel. To keep a safety margin, $3 \mathrm{~W} /$ channel have been considered in the cooling system design. This leads to 


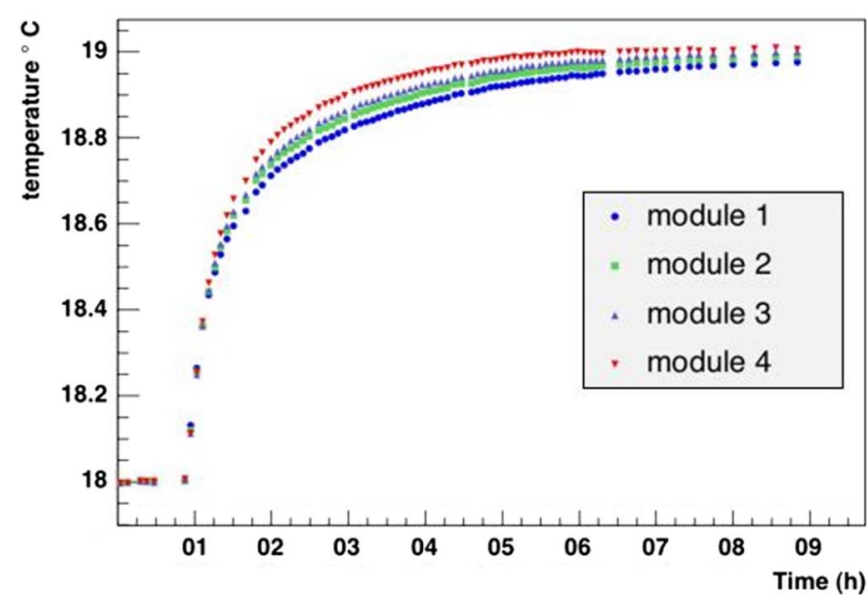

Fig. 1. SM thermal response to $1 \mathrm{~K}$ step on the inlet water temperature. The water temperature step begins at $t=0.5 \mathrm{~h}$. Average measurement of the thermistors glued on the crystals in each module. The different time response between modules can be explained by the weight difference between the modules and by the grid length differences.

a total dissipation of $5100 \mathrm{~W}$ per SM and $184 \mathrm{~kW}$ for the whole ECAL Barrel. The supermodule working temperature has been chosen to be $18^{\circ} \mathrm{C}$ which is the foreseen equilibrium temperature in the experimental cavern and is high enough to avoid any condensation.

\section{CoOling Plant Design}

\section{A. General Requirements on Cooling Plant}

A better way to express the stability requirement on the cooling plant should be that any perturbation on cooling water flow or temperature should not affect the crystal temperature stability by more than $0.05 \mathrm{~K}$ peak to peak. In fact any such perturbation will be filtered by the supermodule thermal inertia. As a consequence the water temperature stability requirement can be put on the temperature averaged over a time interval determined by the supermodule thermal time constant. This time constant has been measured by performing $1 \mathrm{~K}$ temperature step tests (Fig. 1) and found to be about 30 minutes (15 minutes delay between the step beginning and the temperature impact on the thermistors can not be seen on the plot). From this measurement it is clear that small water temperature perturbations (even around $1 \mathrm{~K}$ ) with a time duration below $5 \mathrm{~min}$. have no impact on the crystal's temperature stability.

Assuming $184 \mathrm{~kW}$ total power dissipation and $10 \%$ thermal leak from the electronics, the barrel flow has been set at $50 \mathrm{l} / \mathrm{s}$ at the beginning of the design in order to limit the water temperature increase below $0.1 \mathrm{~K}$ in the volume of the crystals and APDs [3]. Moreover being very conservative and taking into account a water temperature increase between the supermodule water inlet and outlet around $0.9 \mathrm{~K}$, the requirement on the flow stability has been set to $5 \%$ in each supermodule in order to keep the electronic boards temperature variation below $0.05 \mathrm{~K}$.

In conclusion, the requirements on the cooling plant have been expressed as follows.

- The average water temperature over a period of $5 \mathrm{~min}$. should always stay in a range of $0.05 \mathrm{~K}$.
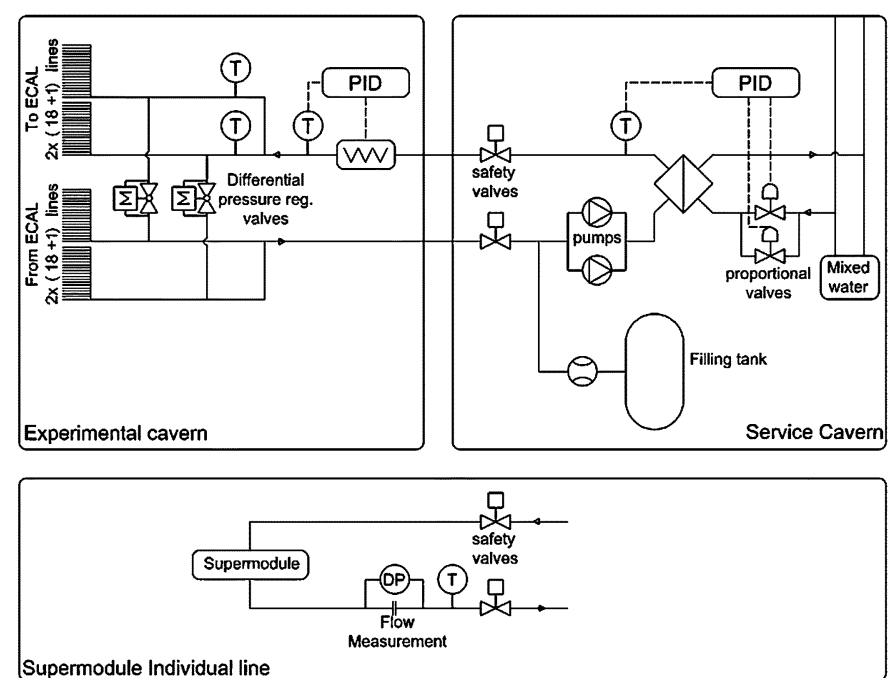

Fig. 2. Final Cooling Plant Schematics. T indicates the location of temperature sensors. PID stands for Proportional Integrate and Derivative represents the PLC performing the regulation. The mixed water is a general purpose cooled water at $14^{\circ} \mathrm{C} \pm 1^{\circ} \mathrm{C}$ available at CMS experiment.

- The flow in each supermodule should always stay at a value of $1.39 \mathrm{l} / \mathrm{s} \pm 2.5 \%$.

- In case one or several supermodules have to be switched off the stability of the temperature and the flow of the other supermodules should stay in the defined range.

- The water temperature at $\mathrm{SM}$ inlet should be $18^{\circ} \mathrm{C} \pm 1^{\circ} \mathrm{C}$.

\section{B. General Design}

The regulation is based on a double stage system (Fig. 2). A rough regulation is performed in a heat exchanger with primary water at $14^{\circ} \mathrm{C} \pm 1^{\circ} \mathrm{C}$ that cools down the water temperature to $T_{\text {exch,set }}$ whilst a more accurate and faster regulation is performed in a second loop by a heater (with $T_{\text {set }}$ as setpoint). Both regulations are performed by PID algorithms. Naming $\delta T_{\text {exch }}$ and $\delta T_{\text {heater }}$ the stability requirements on the primary loop and on the secondary loop (typically $\delta T_{\text {exch }}=$ $0.25 \mathrm{~K}$ and $\delta T_{\text {heater }}=0.025 \mathrm{~K}$ ) and considering that one always needs to keep the primary loop temperature below the set point temperature, the maximum possible exchanger temperature set point is:

$$
T_{\text {exch,set }}=T_{\text {set }}-\delta T_{\text {exch }}
$$

Therefore the heater power has to be set such as to be able to cope with water at a minimal temperature of $T_{\text {exch,set }}-\delta T_{\text {exch }}$. This leads to:

$$
\text { Power }_{\text {heater }}=2 \cdot \delta T_{\text {exch }} \cdot C_{\text {water }} \cdot \text { flow. }
$$

$C_{\text {water }}$ is the water specific heat.

Finally we detail below the requirement that the critical components of the system must fulfill to achieve the required performance.

1) Temperature Sensors: to achieve a $\pm 0.025 K$ stability at the heater outlet the thermal sensors must have a sensitivity better than $0.025 / 5=0.005 K$. The absolute accuracy of the 


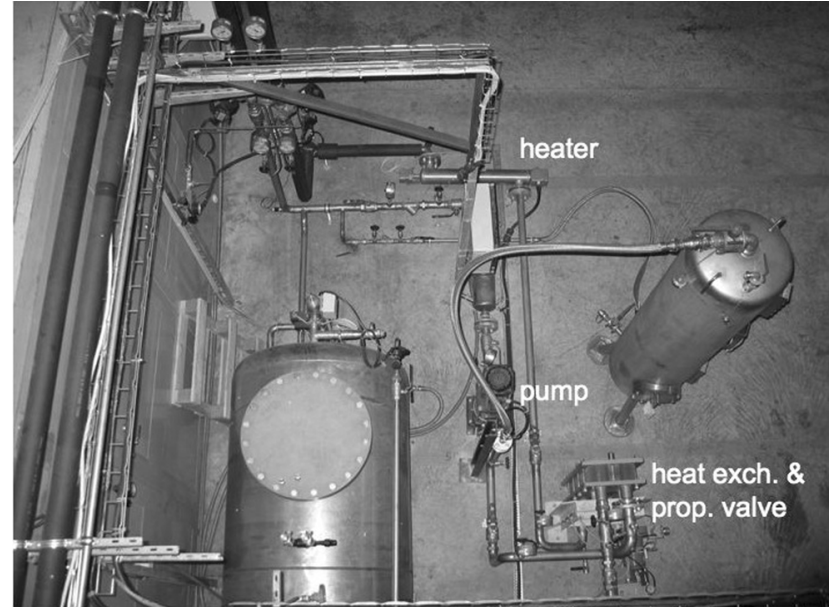

Fig. 3. H4 Cooling Plant picture. This cooling plant is able to cool down 2 SM in parallel. One can see the heat exchanger and the proportional valve on the bottom right, the pump close to the center and the heater at the top of the picture.

sensor is not an issue. The real need is the ability to measure temperature variation.

2) Primary Loop: The exchanger control is performed by regulating the primary water flow. Here the main concern is to choose a valve on which one has a stable and accurate control as it could be the biggest perturbator on all the system.

3) Heater Control: The heater power control accuracy has to be: $\delta T_{\text {exch }} \cdot$ flow $\cdot C_{\text {water }} / 5$. The factor 5 is used to get a good dynamics.

4) Flow Control: The flow is controlled by using differential pressure regulation valves in parallel with the supermodules. As the differential pressure and the pressure drop are fixed, the flow is constant inside each SM even in case of shutting down one or several SM valves. In this last case, the equivalent flow would be immediately diverted through the differential pressure regulation valve.

\section{Prototype Cooling Plant in H4}

At the end of their construction all supermodules are tested at the $\mathrm{H} 4$ test beam area at CERN in order to intercalibrate their channels. Two calibration benches are used, one using cosmic rays while the other one is using an electron beam. The $\mathrm{H} 4$ plant (Fig. 3), which has the same requirements as the final cooling plant except that it is foreseen only for 2 supermodules, has been used to validate and tune the principles of the regulation.

The primary water is provided at a temperature of $14^{\circ} \mathrm{C} \pm 1^{\circ} \mathrm{C}$. The proportional valve to regulate the flow of the primary water at the heat exchanger Inlet is a Masoneiland ${ }^{\mathrm{TM}}$ proportional valve (Fig. 4). The Primary Loop set point is set to $17.95^{\circ} \mathrm{C}$. The heater power is $6 \mathrm{~kW}$. The control of the heater power is performed by thyristors. The temperature sensors are platinum resistance temperature detector (PT100) with transmitter (4-20 mA output in a range of $15^{\circ} \mathrm{C}$ to $25^{\circ} \mathrm{C}$ ) in order to have a high sensitivity and a fast response time $(<2 \mathrm{~s})$. The system is controlled through a SIEMENS ${ }^{\mathrm{TM}}$ serie 200 Programmable Logic Controller (PLC) performing both proportional-integral-derivative (PID) regulations. The general Supervisory Control And Data Acquisition (SCADA) is

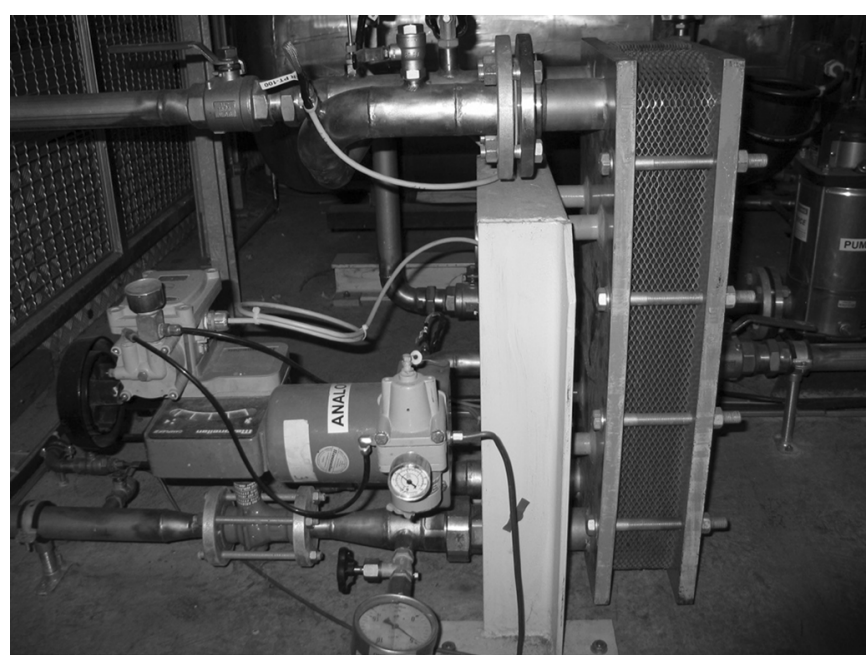

Fig. 4. H4 Cooling Plant Proportional valve and heat exchanger.

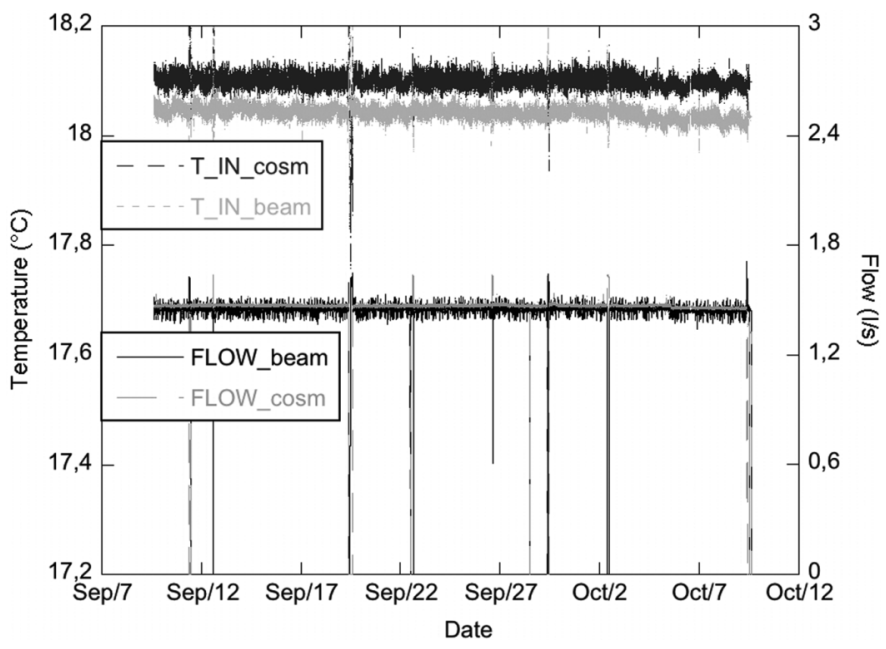

Fig. 5. H4 Cooling Plant Temperature plot: Temperature and flow measurement at the inlet of the 2 supermodules over a period of 1 month.

PVSS $^{\mathrm{TM}}$, which has been chosen as standard for the whole LHC project.

Figs. 5 and 6 show the stability results of the system. Fig. 5 presents the water temperature and flow at the inlet of both SMs. The stability is better than $0.03 \mathrm{~K}$ peak to peak. The perturbations on the water temperature correspond to flow perturbation when the SMs are connected or disconnected from the plant.

Fig. 6 presents the temperature at the exchanger outlet (around $17.95^{\circ} \mathrm{C}$ ) and at the heater outlet (around $18^{\circ} \mathrm{C}$ ). In fact the stability at the exchanger outlet is already better than $0.05 \mathrm{~K}$ peak to peak.

\section{Final Cooling Plant in P5}

The final cooling plant is achieved and is expected to be connected to supermodules by September 2007.

The regulation is performed on the full $62 \mathrm{l} / \mathrm{s}$ flow needed for ECAL ( 50 1/s for the 36 supermodules and 12 1/s for both endcaps). At the heater outlet, the flow is split in 2 and later in 19 parallel lines in order to feed the 36 supermodules and 2 endcaps by independent lines, each equipped with safety isolation valves 


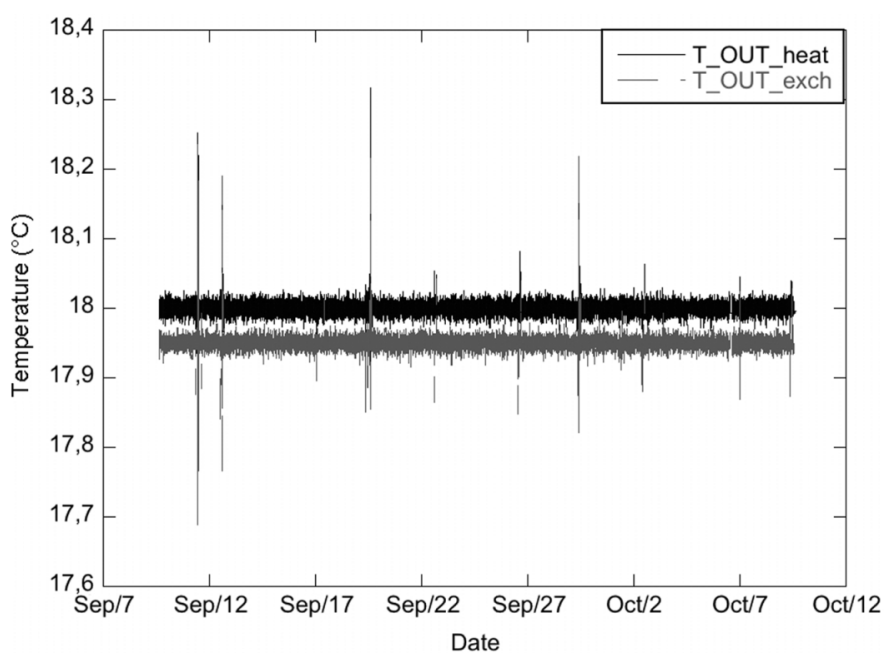

Fig. 6. H4 Cooling Plant Temperature plot: Temperature at the heat exchanger outlet and at the heater outlet.

both at the inlet and outlet side and with flow and temperature measurement at the outlet (see Fig. 2).

In order to have the right sensitivity and stability, 2 proportional valves are installed on the exchanger primary side. One rough regulation valve will be used in open loop while a precise regulation valve will be used for the fine tuning. The temperature stability at exchanger outlet is foreseen to be better than $\pm 0.25 \mathrm{~K}$ (contractual value).

The heater has a power of $150 \mathrm{~kW}$ separated in 9 independent $16.7 \mathrm{~kW}$ lines, each controlled by an independent thyristor. The needed power to heat the $62 \mathrm{l} / \mathrm{s}$ flow by $0.5 \mathrm{~K}(130 \mathrm{~kW})$ corresponds to 8 lines $(133 \mathrm{~kW})$, the ninth being a redundant one. However as the $\mathrm{H} 4$ experience showed a $0.05 \mathrm{~K}$ stability at the primary loop, it has been decided to begin with a reduced power by setting the primary loop to $17.95^{\circ} \mathrm{C}$ and reducing de facto the needed power to $26 \mathrm{~kW}$ - which is a bit less than 2 heater lines. For redundancy purposes 3 thyristors will be used giving a nominal available power of $50 \mathrm{~kW}$.

\section{GENERAL MECHANICS DESIGN}

The mechanics of the cooling inside the supermodule is composed of the regulation cooling and the power cooling. The regulation cooling goal is to keep the crystals and APD's temperature stable whilst the power cooling goal is to remove the power dissipated by the electronics.

\section{A. Regulation Cooling}

It is constructed as a thermal barrier surrounding the crystals (Fig. 7). The regulation in front of the crystals is performed by the thermal screen that is composed of (from the crystals to the front of the supermodule)

- a cylindrical plate: $4 \mathrm{~mm}$ thick aluminum plate for structural reason;

- a first aluminum cooling pipe glued on the cylindrical plate, aluminum chosen to minimize the material density in front of the crystals;

- $3 \mathrm{~mm}$ diameter polyethylene balls (acting as neutron moderator) on $24 \mathrm{~mm}$ thickness;

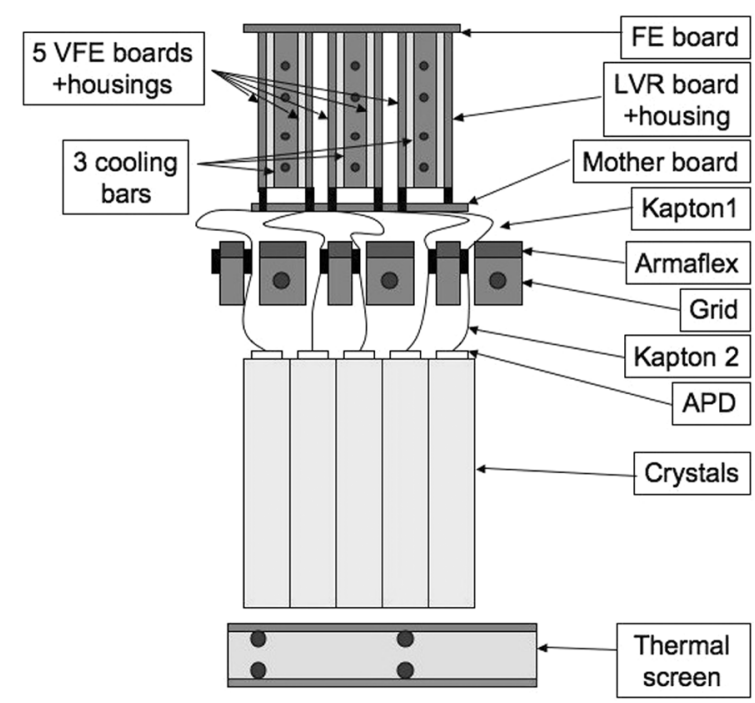

Fig. 7. Cooling mechanics principle.

- A second aluminum cooling pipe (both pipes inside the polyethylene ball volume);

- a $3 \mathrm{~mm}$ thick Rohacell ${ }^{\mathrm{TM}}$ external cover.

On the back, the crystals are fixed on 4 massive aluminum pieces (thickness around $30 \mathrm{~mm}$ ) called the grids. Nine $6 \mathrm{~mm}$ diameter holes have been drilled all along these pieces in order to circulate a high flow of water.

\section{B. Power Cooling}

The electronic read-out chain [6] follows a modular structure whose basic elements are matrices of $5 \times 5$ crystals corresponding to a trigger tower (Fig. 7). Five APD pairs are connected to one Very Front End (VFE) board, and five of these are mounted on one motherboard (MB). Each motherboard is also connected to one Front End (FE) board where the trigger tower primitives are generated. The VFE boards preamplify and shape, then further amplify, and finally digitise the signals from the APDs. Each VFE motherboard also hosts a Low Voltage Regulator board (LVR), which supplies a common voltage of $2.5 \mathrm{~V}$ (required by the $0.25 \mu \mathrm{m}$ IBM CMOS technology) to both the analogue and digital parts of the electronics. Voltage ( $\sim 390 \mathrm{~V}$ ) for the APDs is supplied through the motherboards, with one channel providing a common bias for the 100 APDs of two motherboards. The heat is mainly dissipated in the Low Voltage Regulators and in the VFE boards.

The power from the electronics has to be fully removed without heating the crystals. The main mechanisms of heat exchange between the electronics boards and the crystals are the convection and the conduction through the Kapton cables between the electronics boards and the APDs.

Due to the low APD thermal inertia, the conduction between the VFE boards and the crystals has been the main subject of optimization. To reduce the conduction to the APD, the temperature difference between the APD and the electronics boards has been minimized and the thermal resistance maximized.

Between the grid and the motherboards, a $10 \mathrm{~mm}$ thick layer of insulating foam ( $\mathrm{NH}$ Armaflex ${ }^{\mathrm{TM}}$ ) is placed to minimise the heat flowing towards the crystals by convection. Grid return 


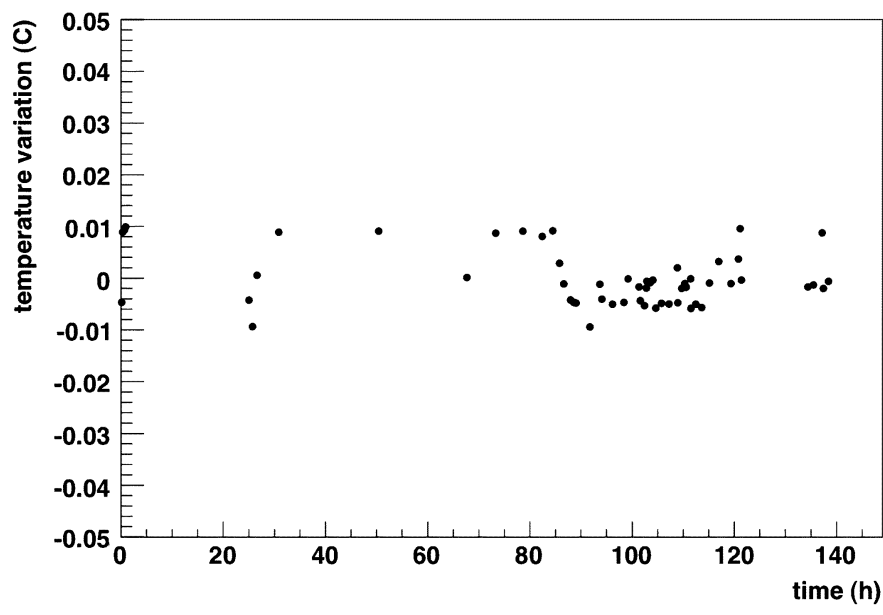

Fig. 8. Temperature variation measurement of a single crystal during SM22 calibration in beam. The stability is about $\pm 0.01 \mathrm{~K}$.

pipes distribute the water through a manifold to a set of aluminium cooling bars. These bars are in close contact with the VFE boards and the LVR boards and have been designed to absorb the heat dissipated by the electronics components mounted on these boards. A thermally conductive paste (gap filler 2000, produced by Bergquist ${ }^{\mathrm{TM}}$ ) is used to provide a good contact between the electronic components and a metal plate facing each board. This plate is coupled to the cooling bar by a conductive pad (ultrasoft gap pad A2000, also produced by Bergquist ${ }^{\mathrm{TM}}$ ).

The connection between the mother boards and the crystals is performed through two kapton cables in series. The length of the kapton between the MB and the grid is $130 \mathrm{~mm}$ while the length of the second kapton is between 70 and $165 \mathrm{~mm}$. The connection between the kapton cables is performed at the grid level (Fig. 7) by connectors that are thermally connected to the grid. Since the thermal resistance between the grid and the connector is much lower than that between the connector and the APD, the major part of the heat goes to the grid - thus to the water circulating in the grid—and not to the APD.

\section{RESULTS}

For one year, $\mathrm{H} 4$ prototype cooling plant has been used to calibrate more than 20 supermodules with cosmic events and 9 with the electron beam. Fig. 8 shows the temperature measurement on an individual crystal during the calibration of one supermodule (SM22). The temperature stability on the considered crystals is $0.02 \mathrm{~K}$ peak to peak. A further analysis of the measurements of all sensors located on the crystals shows a stability at the level of $\pm 0.02 \mathrm{~K}$ within the full supermodule. This stability takes into account all contributors.

Fig. 9 shows the measurement of variation of precision temperature probes located on the thermal screen and grids of the same SM22 during the same period of time. One can also see that the grid and thermal screen temperature stability is better than $\pm 0.02 \mathrm{~K}$.

\section{CONCLUSION}

$\pm 0.05 \mathrm{~K}$ stability over time is a critical requirement to obtain the excellent energy resolution of the crystal calorimeter.

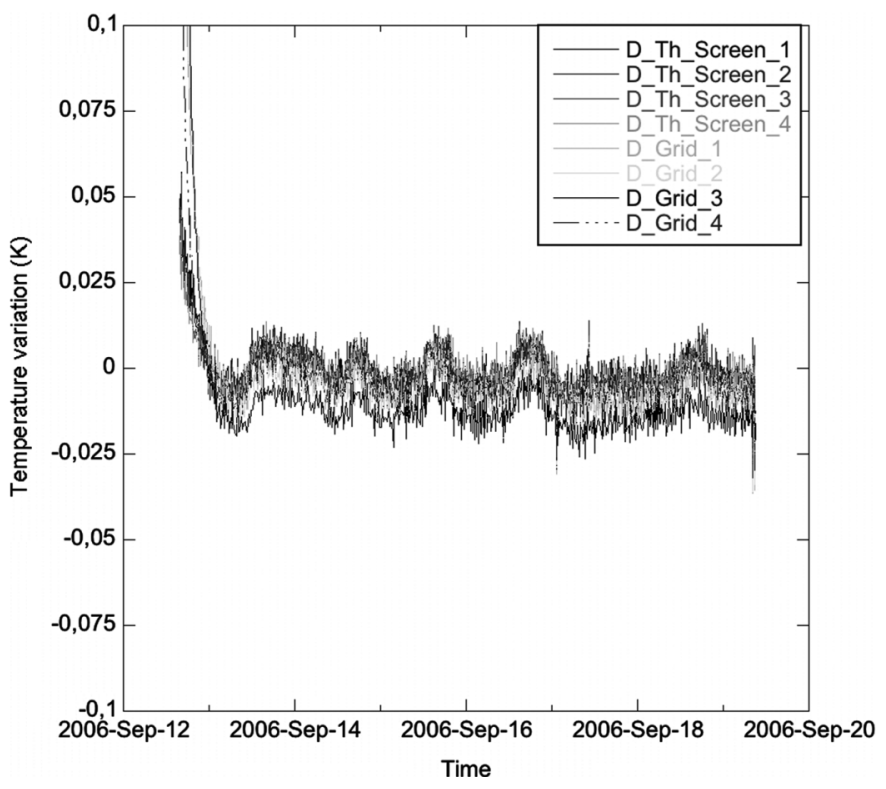

Fig. 9. Temperature variation measurement of the 8 probes of $1 \mathrm{SM}$ during test calibration. The probes are located on the grids and on the inner layer of the thermal shield. One can clearly see a $\sim 0.04 K$ day night effect due to the room temperature variation of about $1 K$.

The development of the cooling mechanics and cooling plant was a 5 years long project involving several physicists and engineers. It required a systematic approach and optimization of the performance at all levels, from the mechanics to the cooling plant design. Innovative solutions have been developed for the electronics cooling. Very precise devices have been used for the cooling plant.

The stability on the crystal temperature during the calibration in $\mathrm{H} 4$ is at the level of $\pm 0.02 \mathrm{~K}$ in full agreement and even exceeding the required performance.

\section{ACKNOWLEDGMENT}

The author would like to thank and acknowledge the large community of technicians and engineers from CERN and collaborating institutes who worked on the design, preparation, and test of the ECAL Cooling System, and in particular X. Pons, G. Peon, P. Pigné, and R. D. Ecclestone, who worked to define and build the $\mathrm{H} 4$ and final cooling plants.

\section{REFERENCES}

[1] The Compact Muon Solenoid Technical Proposal, CERN/LHCC 94-38, 1994, CMS Collaboration.

[2] The Electromagnetic Calorimeter Technical Design Report, CMS TDR 4 CERN/LHCC 97-33, Dec. 15, 1997, CMS.

[3] P. Baillon, "Precise cooling of the ECAL-CMS Calorimeter," in Proc. 8th Int. Conf. Calorimetry in High Energy Physics, Lisbon, Portugal, Jun. 99, pp. 274-283.

[4] P. Govoni et al., "Performances of the cooling system of ECAL CMS," in Proc. 8th ICATPP Int. Conf., Como, Italy, Oct. 6-10, 2003, pp. 203-207.

[5] G. Franzoni et al., "Design and performance of the cooling system for the Electromagnetic Calorimeter of CMS," in Proc. 21st IEEE Instrumentation and Measurement Technology Conf., Como, Italy, May 18-20, 2004, vol. 3, pp. 1861-1865.

[6] Results of the First Performance Test of the CMS Electromagnetic Calorimeter, CMS NOTE 2005/020, Oct. 2005, CMS ECAL Collaboration. 